\title{
PREDICTION OF COMPRESSIVE STRENGTH USING SUPPORT VECTOR REGRESSION
}

\author{
Goutham Sai J, Vijay Pal Singh $\bowtie$ \\ Civil Engineering Department, N.I.T. Kurukshetra, India \\ gsjsai@gmail.com, vpsingh72@gmail.com ${ }^{凶}$
}

\begin{abstract}
At the design stage of a structure, the members of adequate dimension and strength is provided. But with passage of time, the strength of the members reduces gradually due to exposure to environmental conditions and unexpected loadings other than for which the structure is designed. Non Destructive Testing (NDT) method provides a convenient and rapid method of determination of existing strength of concrete without subjecting the member to any damage. In the present study, Support Vector Regression (SVR) in Python has been used for the prediction of compressive strength of concrete. Three different NDT techniques have been used as input for the SVR model. A good co-relation between predicted strength and strength determined after crushing the concrete cubes has been achieved. It has also been observed that accuracy in the predicted strength is more in case of inputs from more than one NDT technique is used.
\end{abstract}

Keywords: rebound hammer, ultrasonic pulse velocity, penetration, support vector

Received: 30 April 2019 regression, NDT.

\section{Introduction}

Concrete is an incredibly essential material in construction and its strength and durability directly influence the stability of structures. The structure must withstand safely all the loads liable to act on them throughout its life. It should also be able to satisfy the serviceability conditions like limitations on deflection and cracking. Hence the concrete used in construction must always have adequate strength and if it undergoes deterioration, it should always be within the limits so that there is no damage to life and property. Therefore, it is very essential to have a system for predicting the strength of material used in existing structures. The testing carried out on existing structures to assess whether the concrete strength is meeting the strength requirement needs to be non-destructive in nature. The various non-destructive testing methods are Rebound hammer test, Ultra sonic pulse velocity etc., where the concrete structure is not damaged except for the nominal damage on the surface to partially destructive test like Core test and Pull out test where the concrete surface has to be slightly repaired after the testing is done. Hence, Non Destructive Testing method has good potential in assessing the integrity of structures throughout its design life without changing or destroying the usefulness of the structure.

The main objective for carrying out NDT is that the various structural properties like strength, quality, inner cracks or voids could be evaluated without causing any damage to the structure. The various NDT methods e.g. Rebound Hammer, Ultrasonic Pulse Velocity and Windsor's probe are used to determine the strength and quality of material in concrete. From the results it has been observed that the strength predicted by different NDT techniques do not confirm with one another. Therefore, the result given by different NDT techniques cannot be used individually for the prediction of concrete strength. It has been observed that to evaluate the strength more accurately, use of more than one method in combination is more appropriate.

The study involves establishing a relationship using SVR in Python between the strength determined by Rebound hammer, Windsor's probe penetration method and Ultrasonic pulse velocity.

\section{Related Works}

There has been increasing demand to test in-situ concrete to estimate the strength. The semi-destructive testing methods involving various techniques are available to overcome the problem of more time, damage of structure due to cutting and extracting of samples. Out of the many available NDT techniques, Schmidt Hammer is the most popular technique because of its ease of operation, less cost and portability. Tests have been conducted to find the reliability of rebound hammer in finding the concrete compressive strength and the variation in the readings due to different exposure conditions. [1]. The total number of samples were divided into 3 groups 
subjected to different environmental conditions. The experiment has revealed that highly dispersive readings are obtained because of variation in surface properties like smoothness of surface and temperature of specimen and though rebound hammer has numerous advantages, the measurements of rebound hammer are not unique. Further studies were conducted to understand the rebound surface hardness of concrete [2]. Experimental results have indicated that Schmidt rebound hammer provides rebound number which is used to establish relationship with concrete compressive strength for high w/c ratio samples i.e. for low strength concrete. On the other hand, for concrete having higher strength, the rebound number is correlated to the Young's modulus of concrete and hence the compressive strength is predicted. Further the concrete cube compressive strength was evaluated using Ultrasonic pulse velocity method [3] and factors affecting the strength were determined. Those factors affecting the strength were also studied for different types of concrete and a simplified expression was defined independent of the nature of concrete and its composition. Further the factors affecting the velocity and strength relationship were also analyzed and artificial neural network was used to model the velocity-strength relationship [4]. It was found out that the aggregates influenced the velocity-strength relationship considerably and it needs to be taken into account for the prediction to be accurate based on ultrasonic pulse velocity. The ANN model developed could predict the concrete compressive strength using pulse velocity as input with satisfactory precision. Studies were also carried out to find a unified relationship between rebound hammer, pulse velocity and compressive strength of concrete [5]. In order to establish the relationship between the NDT values and compressive strength of concrete, regression analysis was carried out and trials were carried out to optimize the correlation equation. Pioneering studies were also carried out to find the influence of material and mix of concrete on rebound hammer and pulse velocity result. Also, the effect of workmanship was also considered in the experiment [6]. Multiple regressions of both the NDT inputs on the crushing strength of concrete generated a set of graphs which provided better prediction of concrete compressive strength. The analysis of in-situ concrete strength assessment was carried out on two RC buildings [7]. The actual concrete strength was assessed by core testing and NDT test were also carried out and its output were used to predict the strength. Single NDT results and as well as their combination has been used for strength prediction. A good quality fit could be established between the predicted and the actual strength. For the prediction of concrete compressive strength ANN has been used [8]. The training of network was done by the modification of weights of connections depending on the information the model has learned. The coefficient of determination was generated to assess how well the model has predicted the strength of concrete. Along with ANN, fuzzy logic has also been used for the concrete compressive strength prediction [9]. The experiments have proved that error rates in fuzzy logic are nominal and hence it can also be successfully used for the prediction of compressive strength of concrete. Artificial Intelligence possesses improved capability over conventional modeling methods. And this superior capability has been used for predicting the strength of HPC using least square support vector regression [10]. For optimization of result, firefly algorithm (FA) has been used which makes the model fast and effective. SVR has achieved the most desirable result with lesser error in prediction.

\section{Experimental Methodology}

Concrete cubes of dimension $150 \mathrm{~mm} \times 150 \mathrm{~mm} \times 150 \mathrm{~mm}$ were used to carry out the experiment. About 350 numbers of concrete cubes of above dimensions with varying composition of cement, sand, aggregate and watercement ratios were casted and tested in the laboratory. The concrete cubes were cured under controlled temperature of $25 \pm 2^{\circ} \mathrm{C}$ and tested after 28 days. Each sample of cube was tested initially by above mentioned NDT methods. Finally, the samples were tested under compression testing machine for obtaining actual compressive strength.

\section{Analytic Study Using Support Vector Regression}

Support Vector Machines (SVM) are widely used machine learning techniques for classification problems. Regression methods are also used in SVM, by keeping all the main parameters that characterize the algorithm. In support vector regression (SVR), the aim is to determine the function which is unknown-continuously valued based on a finite number set of input samples [11].

The loss function used in SVR is a new function called insensitive loss function, in which the absolute residual is off target by or less, then no penalty is assigned.

$$
\mathrm{L}_{\varepsilon}(\mathrm{y}, \mathrm{f}(\mathrm{x}, \omega))=\left\{\begin{array}{cc}
0 & \text { if }|y-f(x, \omega) \leq \varepsilon| \\
\mid y-f(x, \omega)-\varepsilon & \text { otherwise }
\end{array}\right.
$$




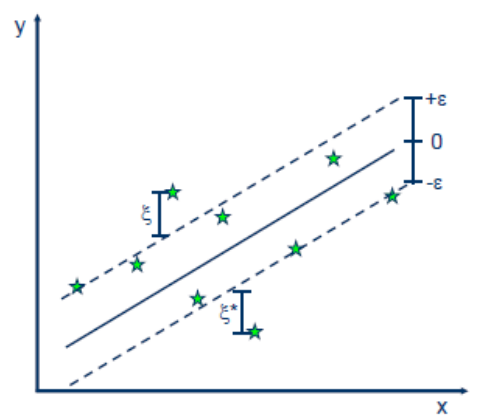

Figure 1: Linear SVR for tube fitting with radius $\varepsilon$ to the data [12]

Fig. 1 shows a linear SVR where linear regression is performed using the new loss function and simultaneously reduces the complexity of model by minimizing $\|\omega\|^{2}$. This can be explained by defining (nonnegative) slack variables $\xi_{i}, \xi_{i}^{*}, i=1, \ldots n$, for the measurement of deviation in training samples outside $\varepsilon$-insensitive zone. The optimization problem can be deduced as a minimization problem with the following objective function.

$$
\begin{gathered}
\text { Min } \frac{1}{2}|| \omega \|^{2}+C \sum_{i=1}^{n}\left(\xi_{i}+\xi_{i}^{*}\right) \\
\text { Subject to }\left\{\begin{array}{l}
y_{i}-f\left(\boldsymbol{x}_{\boldsymbol{i}}, \omega\right) \leq \varepsilon+\xi_{i}^{*} \\
f\left(\boldsymbol{x}_{\boldsymbol{i}}, \omega\right)-y_{i} \leq \varepsilon+\xi_{i} \\
\xi_{i}, \xi_{i}^{*} \geq 0, i=1, \ldots, n
\end{array}\right.
\end{gathered}
$$

The constant $C$ in the above equation finds out the penalties in errors of estimation. Higher value of $C$ imposes a higher value of penalty to errors such that the training of regression is done to reduce the error with lesser generalization. When the value of $C$ is less, it imposes lower value of penalty to errors. This leads to the margin minimization with errors. The ability for generalization becomes higher. The optimization problem in the above equation can be converted to dual problem. Its solution is given by

$$
f(\boldsymbol{x})=\sum_{i=1}^{n_{S V}}\left(\alpha_{i}-\alpha_{i}^{*}\right) K\left(\boldsymbol{x}_{i}, \boldsymbol{x}\right)
$$

where $0 \leq \alpha_{i}^{*} \leq C, 0 \leq \alpha_{i} \leq C$, where $f(\boldsymbol{x})$ is the estimated function, $\alpha_{i}$ and $\alpha_{i}^{*}$ are Lagrange multipliers corresponding to the two constraints in (2). $n_{S V}$ denotes the number of Support Vectors $(\mathrm{SVs}) . K\left(\boldsymbol{x}_{\boldsymbol{i}}, \boldsymbol{x}\right)$ which is the kernel function, converts the data into a space of higher dimension. This transformation allows to perform a linear separation. The radial basis function $(\mathrm{RBF})$ is commonly used as the kernel for regression:

$$
k\left(x_{i}, x\right)=\exp \left\{-\gamma\left|x-x_{i}\right|^{2}\right\}
$$

The performance of SVR depends on fine tuning of parameters $C, \varepsilon$ and the kernel parameters like $\gamma$. The values of these parameters adopted in this study is based on trial and error method.

\section{Results and Discussion}

The data obtained from experimental study has been analyzed using support vector regression. 7 models were developed out of which 3 models had each of the NDT technique output as input. Another 3 were modelled with combination of two NDT technique output as input. And the last model had the output of all the three NDT technique as input. In all the models, the target value provided was the actual compressive strength of concrete which was found out by subjecting the specimens to compressive load upto failure. Curves as shown in Fig. 2-4 were developed which shows the relationship between the concrete compressive strength and NDT data.

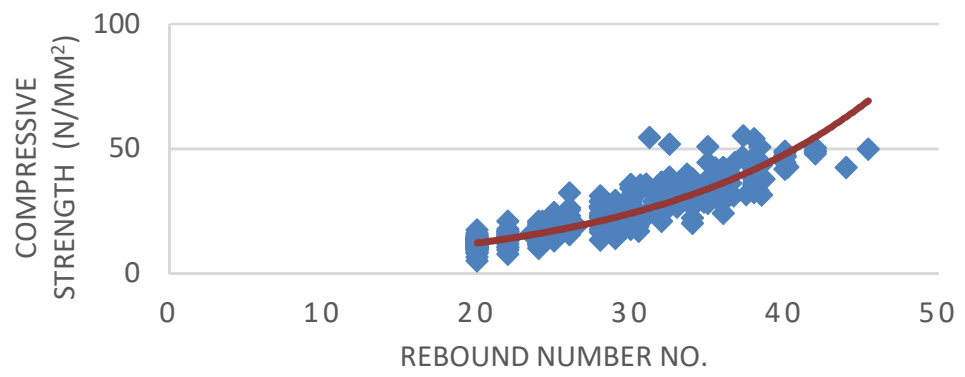

Figure 2: Relationship Rebound Hammer No. and Actual compressive strength. 


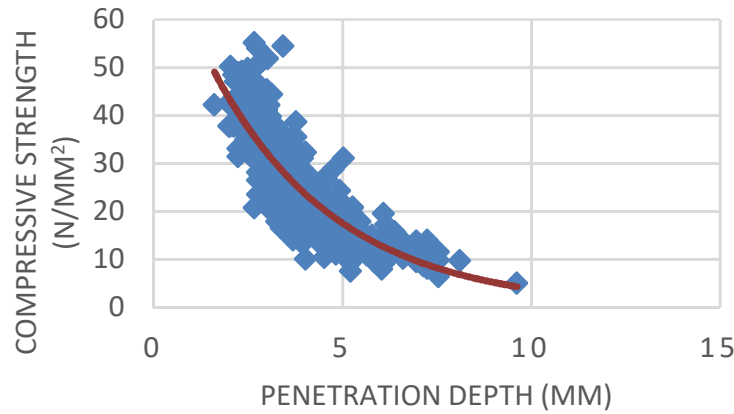

Figure 3: Relationship Penetration Depth. and Actual compressive strength.

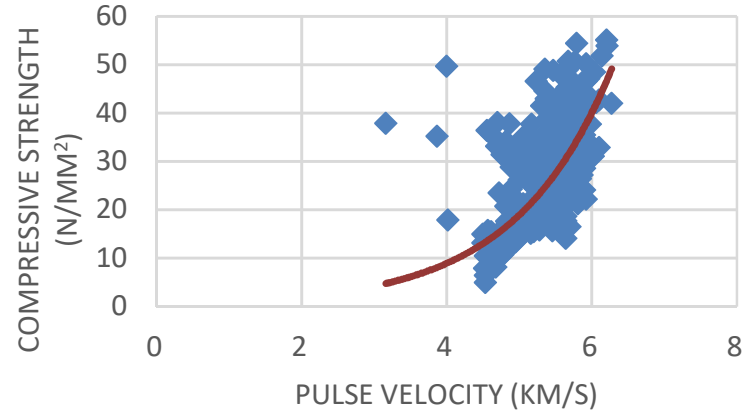

Figure 4: Relationship Pulse Velocity and Actual compressive strength.

Various kernel functions like linear, polynomial, rbf and sigmoid were used and out of these rbf kernel function yielded the most precise output. Out of the total data, $70 \%$ was used for training and $30 \%$ for testing.

Comparison is made between the strength predicted by SVR model and the actual compressive strength and is shown in Fig. 5-11. Fig. 5-7 shows the relationship between the strength predicted by individual NDT techniques and the actual compressive strength. Coefficient of determination (R2) was determined for the prediction made by NDT techniques individually and as well as for their combination. For predictions from individual NDT techniques, the coefficient of determination (R2) is highest for rebound hammer prediction, followed by penetration depth and pulse velocity prediction.

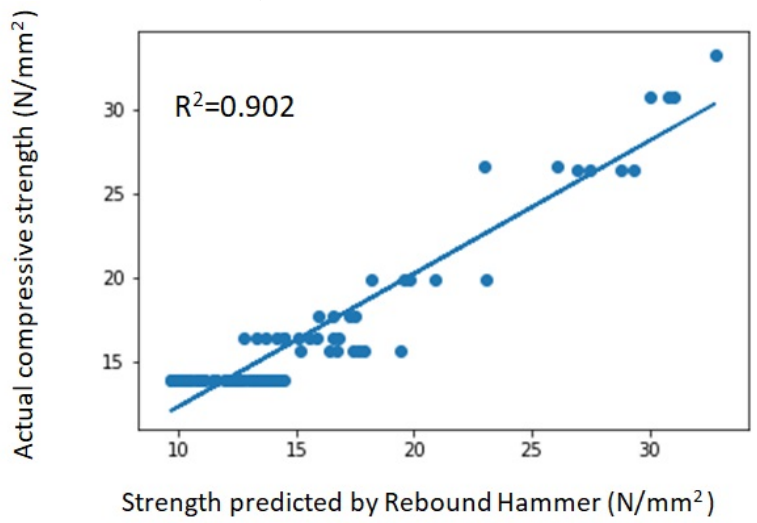

Figure 5: Relationship between strength predicted by Rebound Hammer and actual compressive strength.

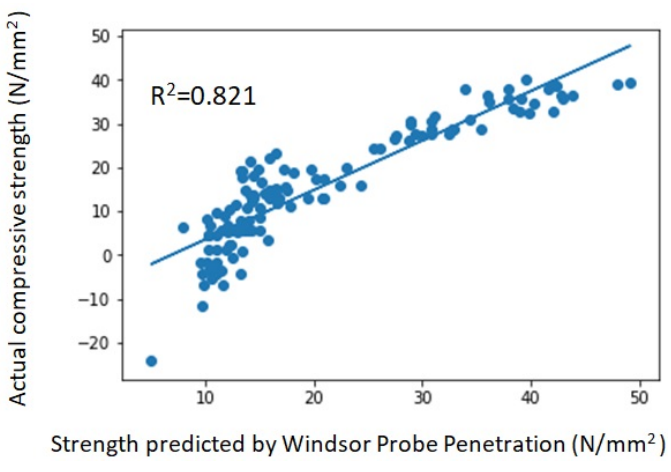

Figure 6: Relationship between strength predicted by Probe Penetration and actual compressive strength.

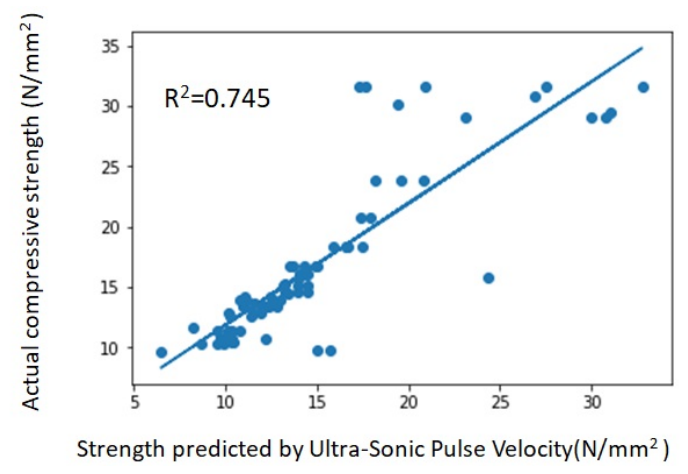

Figure 7: Relationship between strength predicted by Pulse Velocity and actual compressive strength.

Fig. 8-10 depicts the relationship between the compressive strength predicted by combining the outputs of 2 NDT techniques and the actual concrete compressive strength. Highest level of precision in the prediction is given by Pulse Velocity-Rebound Hammer combination, followed by Penetration Depth-Pulse Velocity 
Combination and Rebound Hammer-Penetration Depth combination. Fig. 11 depicts the relationship between the strength predicted by combining the output of all the 3 NDT techniques and the actual compressive strength.

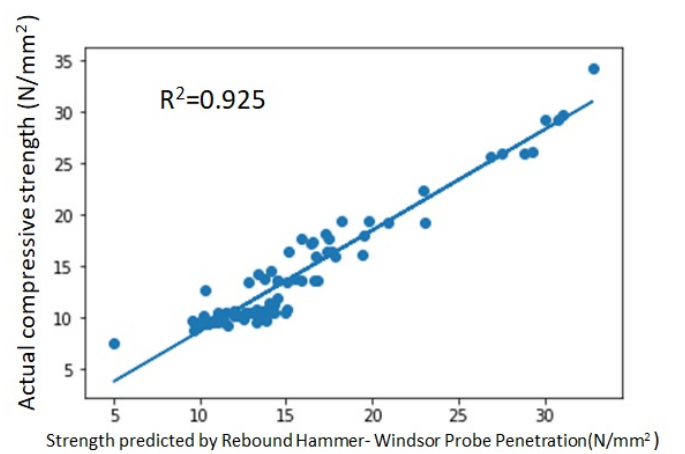

Figure 8: Relationship between strength predicted by Rebound Hammer-Probe Penetration and actual compressive strength.

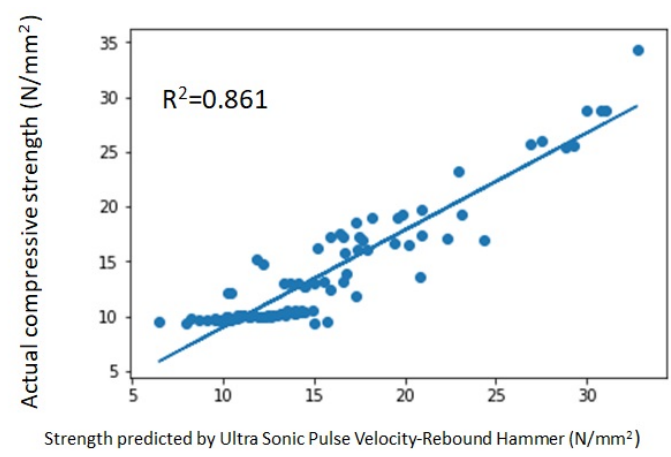

Figure 10: Relationship between strength predicted by Pulse Velocity, Rebound Hammer and Crushing compressive strength.

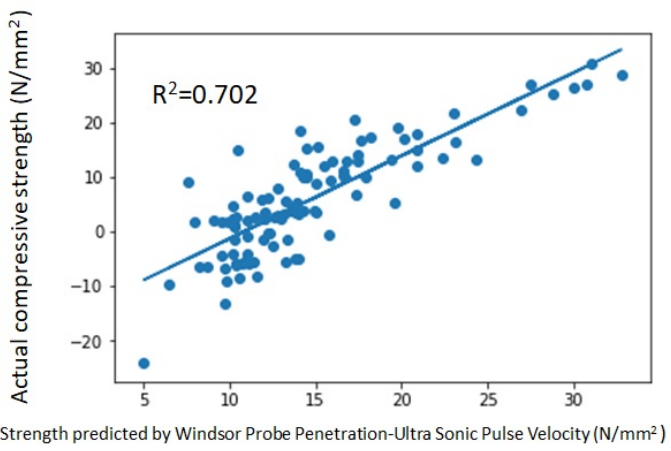

Figure 9: Relationship between strength predicted by Probe Penetration, Pulse Velocity and crushing compressive strength.

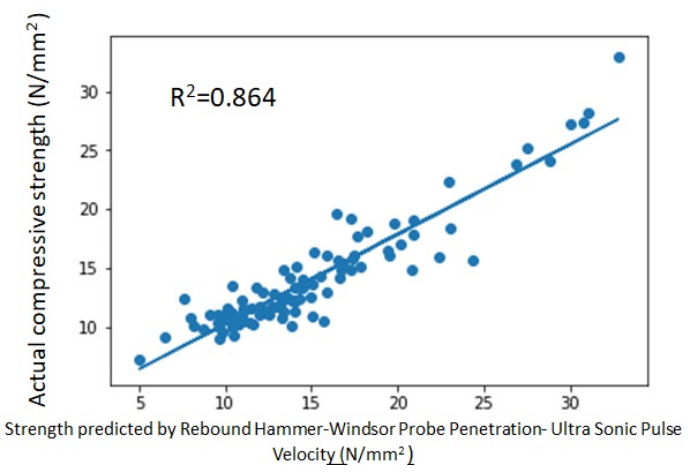

Figure 11: Relationship between strength predicted by Rebound Hammer- Probe Penetration-Pulse Velocity and actual compressive strength.

Though strength prediction using Pulse velocity alone doesn't yield good result, when it is used in combination with Probe Penetration and Rebound Hammer, the results obtained are of very high accuracy. The coefficient of determination $\left(\mathrm{R}^{2}\right)$ for individual NDT techniques and as well as for their combination is shown in Table 1.

Table 1: NDT Techniques v/s Coefficient of determination

\begin{tabular}{cll}
\hline S. No. & Name of Technique/Method & $\mathbf{R}^{\mathbf{2}}$ \\
\hline 1 & Rebound Hammer & 0.902 \\
2 & Windsor' Penetration & 0.821 \\
3 & Ultra Sonic pulse velocity & 0.745 \\
4 & Rebound Hammer-Windsor' Penetration & 0.925 \\
5 & Windsor' Penetration- Ultra Sonic pulse velocity & 0.702 \\
6 & Ultra Sonic pulse velocity-Rebound Hammer & 0.8612 \\
7 & Rebound Hammer-Windsor' Penetration-Ultra Sonic pulse velocity & 0.8647 \\
\hline
\end{tabular}

From Table 1, it has been observed that the coefficient of determination $\left(\mathrm{R}^{2}\right)$, which determines the closeness to the actual result is comparatively less for Ultra sonic pulse velocity method when compared to the other two NDT techniques. Hence this method alone cannot be used to determine the concrete compressive strength as its prediction will yield poor result. But it can be used in combination with Rebound Hammer since the coefficient of determination is higher when combined. The prediction results when all the 3 NDT techniques are used in combination is high, but the best prediction is given by Rebound Hammer-Windsor' Penetration. Its coefficient of determination is very much higher and hence its accuracy of prediction will also be high. Hence the predicted results will have minimal error. 


\section{Conclusion}

The present study shows that SVR can be used for the prediction of concrete compressive strength and it has been observed that the predictions are very much close to the actual compressive strength. Further, the accuracy of prediction can be increased by providing different combinations of NDT output as data to the SVR model. From the study it has been concluded that:

- In the case of single NDT technique, the strength prediction using Rebound Hammer is the most reliable method. It has a very good coefficient of determination of 0.902 . This makes the judgement using rebound hammer quite easy and reliable.

- The strength prediction using Probe Penetration alone yields a lower coefficient of determination of 0.821 and the accuracy decreases further when it comes to prediction using pulse velocity method, whose coefficient determination is 0.745 . Hence these methods cannot be used alone to predict the compressive strength of concrete.

- When the output of 2 NDT techniques were used in combination and fed to the SVR model, higher value of coefficient of determination of 0.925 could be achieved for Rebound Hammer- Windsor Penetration combination. Though pulse velocity cannot be used alone for strength prediction, it can be used in combination with Rebound Hammer and reliable predictions can be achieved, since its coefficient of determination is 0.861 .

- When 3 NDT techniques were used in combination, coefficient of determination of 0.864 could be achieved which is appreciable. But the best prediction model in SVR is Rebound Hammer- Windsor' Penetration combination.

\section{References}

[1] Sanchez, K. and Tarranza, N. 2014. Reliability of Rebound Hammer Test in Concrete Compressive Strength Estimation. International Journal of Advances in Agricultural \& Environmental Engineering 1, 2, pp. 198-202.

[2] Szilágyi, K., Borosnyói, A., and Zsigovics, I. 2015. Understanding the rebound surface hardness of concrete. Journal of Civil Engineering \& Management 21, 2, pp. 185-192.

[3] Bogas, J. A., Gomes, M. G., and Gomes, A. 2013. Compressive strength evaluation of structural lightweight concrete by non-destructive ultrasonic pulse velocity method. Ultrasonics 53, 5, pp. $962-972$.

[4] Trtnik, G., Kavcic, F., and Turk, G. 2009. Prediction of concrete strength using ultrasonic pulse velocity and artificial neural networks. Ultrasonics 49, 1, pp. 53-60.

[5] Nash't, I. H., A'bour, S. H., and Sadoon, A. A. 2005. Finding an Unified Relationship between Crushing Strength of Concrete and Non-destructive Tests. In Middle East Nondestructive Testing Conference \& Exhibition. 27-30 Nov 2005 Bahrain, Manama.

[6] Jain, A., Kathuria, A., Kumar, A., Verma, Y. and Murari, K. 2013. Combined Use of Non-Destructive Tests for Assessment of Strength of Concrete in Structure. Procedia Engineering 54, pp. 241-251.

[7] Benyahia, K. A., Sbartaï, Z. M., Breysse, D., and Ghrici, S. K. M. 2017. Analysis of the single and combined non-destructive test approaches for on-site concrete strength assessment: General statements based on a real case-study. Case Studies in Construction Materials 6, pp.109-119.

[8] Yeh, I. C. 1998. Modeling Of Strength Of High-Performance Concrete Using Artificial Neural Networks. Cement and Concrete Research 28, 12, pp. 1797-1808.

[9] Topcu,I. B. and Sarıdemir, M. 2008. Prediction of compressive strength of concrete containing fly ash using artificial neural networks and fuzzy logic. Computational Materials Science 41, 3, pp. 305-311.

[10] Pham, A. D., Hoang, N. H. and Nguyen, Q. T. 2015. Predicting Compressive Strength of High-Performance Concrete Using Metaheuristic-Optimized Least Squares Support Vector Regression. Journal of Computing in Civil Engineering 30, 3, No. 06015002.

[11] Vapnik, V. N. 1995. The Nature of Statistical Learning Theory. Springer, New York.

[12] Vapnik, V. N. 1999. An overview of Statistical Learning Theory. IEEE Transactions on Neural Networks 10, 5, pp. 988-999. 